\title{
Higher-derivative gravity in string theory
}

\author{
Kristin Förger ${ }^{\text {a }}$, Burt A. Ovrut ${ }^{b}$, Stefan J. Theisen ${ }^{a}$, Daniel Waldram ${ }^{b}$ \\ a Sektion Physik, Universität München, Theresienstr. 37, 80333 München, Germany \\ ${ }^{b}$ Department of Physics, University of Pennsylvania, Philadelphia, PA 19104-6396, USA \\ Received 25 July 1996 \\ Editor: M. Dine
}

\begin{abstract}
We explicitly extract the structure of higher-derivative curvature-squared terms at genus 0 and 1 in the $d=4$ heterotic string effective action compactified on symmetric orbifolds by computing on-shell $S$-matrix superstring amplitudes. In particular, this is done within the context of calculating the graviton 4-point amplitude. We also discuss the modulidependent gravitational threshold corrections to the coupling associated with the $C P$ even quadratic curvature terms.
\end{abstract}

\section{Introduction}

It is a well known fact that perturbative string theory contains the pure Einstein-Hilbert action. A priori, there is no reason why higher-derivative gravitational terms could not also be present in the action of strings and superstrings. Recently, it has been shown that higher-derivative supergravity terms can provide a new mechanism for supersymmetry breaking in $d=2$ and $d=4$ supergravity models [3] and, hence, such terms could be fundamentally important. An attempt to determine this question was made within the context of superstring compactifications on $Z_{N}$ symmetric orbifolds in [4]. It was demonstrated, using light field one-loop radiative corrections to the string effective Lagrangian, that higher-derivative supergravitational terms can arise in string theory and, importantly, that they need not be in the topological Gauss-Bonnet combination. However, this discussion was not conclusive since the radiative contributions of the infinite tower of massive states was not included in the calculation. It is possible, although it was argued that it was unlikely, that these massive contributions would can- cel the higher-derivative terms generated by the light fields. It seems clear that the only way to resolve this issue is to do complete genus- 0 and genus- 1 superstring amplitude calculations, since such calculations include both massless and massive states. One would then attempt to construct the string action associated with these amplitudes. In this way one could explore the interesting question of whether or not string theory can completely fix the curvature squared terms in its action, at least to the genus- 1 level. The answer to this problem is the subject of this paper.

\section{String $S$-matrix}

The perturbative $S$-matrix approach consists of finding a local gauge-invariant effective Lagrangian $\mathcal{L}$ whose associated $S$-matrix elements coincide with the string $S$-matrix. More precisely this means that the effective action, which is a power series in the external momenta $k^{2} \alpha^{\prime}$ and the string coupling constant $g_{s}$, reproduces the kinematic structure of the string amplitudes. 
We start by giving the most general ansatz for the bosonic part of the $C P$ even gravitational Lagrangian

$$
\begin{aligned}
\mathcal{L} & =\sqrt{g}\left\{\frac{1}{2 \kappa^{2}} R+a R_{\mu \rho \nu}{ }^{\sigma} R^{\mu \rho \nu}{ }_{\sigma}\right. \\
& \left.+b R_{\mu \nu} R^{\mu \nu}+c R^{2}\right\}
\end{aligned}
$$

where $\kappa$ is the four dimensional gravitational coupling constant. In the following we set $2 \kappa^{2}=1$ and reintroduce it later for special purposes. We are not considering the $C P$-odd part of the gravitational action here, which is given by a topological invariant, the first Pontrjagin class in four dimensions. The aim is to try to fix the unknown coefficients $a, b$ and $c$ by calculating string amplitudes.

In order to find the appropriate $n$-point string amplitudes, we expand the above Lagrangian around the flat background metric $g_{\mu \nu}=\eta_{\mu \nu}+h_{\mu \nu}$. The inverse metric is then given by a power series expansion $g^{\mu \nu}=$ $\eta^{\mu \nu}-h^{\mu \nu}+\left(h^{2}\right)^{\mu \nu}-\ldots$ and $h^{\mu \nu}=\eta^{\mu \rho} \eta^{\nu \sigma} h_{\rho \sigma}$. Furthermore, in order to correspond to the on-shell fluctuations described by the string graviton-vertex operator, we demand that $h_{\mu \nu}$ satisfies the harmonic gauge conditions for a massless spin 2 field, namely $\square h_{\mu \nu}=$ $0, \partial^{\mu} h_{\mu \nu}=0$ and the tracelessness condition $h=0$. Expanding the curvature terms in Eq. (1) in terms of $h$, the first non-vanishing contributions arise at the 3point level [7]. These are

$$
\begin{aligned}
& \left.\sqrt{g} R\right|_{h^{3}}=h_{\mu \nu}\left(h^{\mu \nu} h\right)+2 h_{\mu \nu},{ }^{\sigma} h^{\mu \rho},^{\mu} h_{\rho \sigma} \\
& \quad \rightarrow\left(k_{2} \epsilon^{1} k_{2}\right)\left(\epsilon^{2} \epsilon^{3}\right)+2\left(k_{3} \epsilon^{2} \epsilon^{3} \epsilon^{1} k_{2}\right) \\
& \quad+\text { cyclic perm } .
\end{aligned}
$$

$$
\begin{aligned}
& \left.\sqrt{g} R_{\mu \rho \nu}{ }^{\sigma} R_{\sigma \rho \nu}^{\mu \nu}\right|_{h^{3}}=h_{\mu \nu},{ }^{\rho \lambda} h_{\rho \sigma} h^{\sigma \lambda},{ }^{\mu \nu} \\
& \quad \rightarrow\left(k_{1} \epsilon^{3} k_{1}\right)\left(k_{3} \epsilon^{2} \epsilon^{1} k_{3}\right)+\text { cyclic perm. }
\end{aligned}
$$

where the notation $\left(h^{\mu \nu} h\right)=\left(\partial^{\mu} \partial^{\nu} h_{\rho \sigma}\right) h^{\rho \sigma}$ has been used. We have also introduced the transformation to the momentum space, replacing $i \partial \rightarrow k$, and replaced $h_{\mu \nu} \rightarrow \epsilon_{\mu \nu}$, where $\epsilon_{\mu \nu}$ is the graviton polarization tensor. Note that this polarization tensor satisfies the same differential and tracelessness conditions as $h_{\mu \nu}$. Unfortunately, the kinematic structure of $R_{\mu \nu} R^{\mu \nu}$ and $R^{2}$ are such that they must vanish when expanded in $h_{\mu \nu}$ to the 3-point level. That is

$\left.\sqrt{g} R_{\mu \nu} R^{\mu \nu}\right|_{h^{3}}=\left.\sqrt{g} R^{2}\right|_{h^{3}}=0$
Therefore, a 3-point string amplitude with three onshell external gravitational vertex operators can produce only the first two terms of the effective Lagrangian $\mathcal{L}$. The other two terms may exist in the effective Lagrangian, but this cannot be determined at the 3-point level. Fortunately, at the level of 4-point amplitudes (and higher), the Ricci squared and curvature scalar squared terms no longer vanish. That is, at the level of 4-point amplitudes $\left.\sqrt{g} R_{\mu \nu} R^{\mu \nu}\right|_{h^{4}}$ and $\left.\sqrt{g} R^{2}\right|_{h^{4}}$ are non-zero. In the expansion up to order $h^{4}$ of the curvature squared terms, it is sufficient for our purposes to isolate only certain terms, which are particular combinations of the polarization tensors, namely

$$
\begin{aligned}
& \left.\sqrt{g} R_{\mu \rho \nu}{ }^{\sigma} R^{\mu \rho \nu}{ }_{\sigma}\right|_{h^{4}}=\left(\frac{1}{8} u^{2}+\frac{3}{2} s^{2}\right) E_{2} \\
& \quad+\left(\frac{1}{8} t^{2}-\frac{1}{4} s^{2}\right) E_{1}+\ldots \\
& \left.\sqrt{g} R_{\mu \nu} R^{\mu \nu}\right|_{h^{4}}=\frac{1}{4} s^{2} E_{2}+\left(\frac{1}{16} t^{2}+\frac{1}{8} s^{2}\right) E_{1}+\ldots \\
& \left.\sqrt{g} R^{2}\right|_{h^{4}}=\frac{9}{16} s^{2} E_{1}+\ldots
\end{aligned}
$$

where $s=-2 k_{1} \cdot k_{2}, t=2 k_{1} \cdot k_{3}$ and $u=-2 k_{1} \cdot k_{4}$ are the Mandelstam variables and we introduce $E_{1}=$ $\left(\epsilon_{1} \epsilon_{2}\right)\left(\epsilon_{3} \epsilon_{4}\right)$ and $E_{2}=\left(\epsilon_{1} \epsilon_{2} \epsilon_{4} \epsilon_{3}\right)$, using the matrix notation $\left(\epsilon_{1} \epsilon_{2}\right)=\epsilon_{1 \mu \nu} \epsilon_{2}{ }^{\mu \nu}$.

At tree level, both the 3- and 4-point graviton amplitudes for the heterotic string in $d=4$ have already been studied in, e.g. $[7,12,17,8]$. The $\mathcal{O}\left(k^{2}\right)$ part of the 3-point tree level amplitude corresponds to the curvature scalar $R$ and, therefore, reproduces the Einstein-Hilbert action. Additionally, it gives the relation $2 \kappa^{2}=g_{s}^{2} \alpha^{\prime}$ between the gravitational and string coupling constants. The $\mathcal{O}\left(k^{4}\right)$ terms in the 3-point amplitudes can only give rise to one of the curvature squared terms, as discussed above, so we won't discuss them here. All three curvature squared terms arise at order $\mathcal{O}\left(k^{4}\right)$ in the four-graviton amplitude. If we again restrict ourselves to only the terms involving the particular polarization combinations $E_{1}$ and $E_{2}$, the $\mathcal{O}\left(k^{4}\right)$ contribution of the 4-point amplitude is found to be

$$
\begin{aligned}
\mathcal{A}_{4 g}^{\text {tree }} & =3 g_{s}^{2}\left\{\left(-\frac{1}{2} s^{2}+t^{2}\right) E_{1}+u^{2} E_{2}\right\} \\
& \equiv 3 g_{s}^{2} K_{4 g}^{\text {tree }}
\end{aligned}
$$


Comparing this amplitude with the effective Lagrangian $\mathcal{L}$, one realizes that there is no combination of curvature squared terms (5), (6) and (7) that reproduces the kinematic structure of $\mathcal{A}_{4 g}^{\text {tree }}$. This fact is not surprising since string amplitudes include $1 P R$ exchange graphs with massless poles as well as 1PI graphs. It follows that one has to perform the appropriate field theory subtractions in order to relate the string result to the effective action. This will be done for the tree and the 1-loop amplitudes in the next section.

Proceeding in a similar fashion to the genus-zero case, we now go to the one-loop level and calculate both the 3-and 4-point graviton amplitudes on a worldsheet torus for the heterotic string in $d=4$ with a given vacuum. The general expression for the $C P$ even $n$-point amplitude is $[13,15]$

$$
\begin{gathered}
\mathcal{A}_{n}^{\text {even }}=g_{s}^{n} \sum_{\substack{\left(s_{1}, s_{2}\right) \\
\text { even }}}(-)^{s_{1}+s_{2}} \int_{\tau \in \Gamma} \frac{d^{2} \tau}{\tau_{2}} Z(\tau, \bar{\tau}, s) \\
\times \int \prod_{i=1}^{n} d^{2} z_{i}\left\langle\prod_{i=1}^{n} V^{(0)}\left(z_{i}, \bar{z}_{i}\right)\right\rangle_{s}
\end{gathered}
$$

where $s=\left(s_{1}, s_{2}\right)$ characterizes the spin structures, which take the values 0 and 1 for the NS and R sectors, respectively. The integration region for the modulus of the torus $\tau=\tau_{1}+i \tau_{2}$ is the fundamental region $\Gamma=\left\{\tau|| \tau_{1}\left|\leq \frac{1}{2},\right| \tau \mid \geq 1\right\}$. The factor $(-)^{s_{1}+s_{2}}$ comes from the fermionic partition function $Z_{u t}$ in the light cone gauge by demanding that the sum over the spin structures of $Z_{\psi}$ is modular invariant. $Z(\tau, \bar{\tau}, s)$ is the partition function in light cone gauge for the heterotic string, given by

$$
\begin{aligned}
& Z(\tau, \bar{\tau}, s)=\operatorname{Tr}\left((-)^{s_{2} F} q^{H-\frac{1}{2}} \bar{q}^{\tilde{H}-1}\right) \\
& \quad=Z_{\psi} Z_{X} Z_{X_{0}} Z_{\text {int }}
\end{aligned}
$$

where $F$ is the fermion number and $q=e^{2 \pi i \tau}, Z_{X_{0}}=$ $1 / 2(2 \pi)^{4} \gamma_{2}^{2}$ is the contribution from the bosonic zero modes, $Z_{X}=\frac{1}{|\eta(\tau)|^{4}}$ is the bosonic partition function where the Dedekind $\eta$ function is defined as $\eta(\tau)=$ $q^{1 / 24} \prod_{n=1}^{\infty}\left(1-q^{n}\right)$, and the partition function for one complex fermion in the light cone gauge is $Z_{\psi}=$ $\frac{\vartheta_{\alpha}(0, \tau)}{\eta(\tau)}$ where $\vartheta_{\alpha}$ are the Riemann theta functions for $\alpha=2,3,4$ corresponding to the spin structures $\left(s_{1}\right.$, $\left.s_{2}\right)=(1,0),(0,0),(0,1)$ respectively. The term $Z_{\text {int }}$ is the partition function for the six-dimensional inter- nal compact manifold. The super-ghost charges in the $C P$ even part of the string amplitude have to add up to zero. Therefore, the vertex operators are taken to be in the zero ghost picture $V^{(0)}$. In this picture, the graviton vertex operator looks like

$$
V_{g}^{(0)}(z, \bar{z})=: \epsilon_{\mu \nu} \bar{\partial} X^{\mu}\left(\partial X^{\nu}+i k \cdot \psi \psi^{\nu}\right) e^{i k \cdot X}:
$$

Here $X^{\mu}$ are the free world-sheet bosons and $\psi^{\mu}$ the left-moving supersymmetric partners.

Let us start by calculating the $\mathcal{O}\left(k^{2}\right)$ piece of the 3-point graviton amplitude, which is associated to the $R$ term. We expand the three-point correlation function of three-graviton vertex operators in the zero ghost picture using Wick contractions. By supersymmetry, terms that are independent of the spin structure give zero after summing over spin structures. Contractions of the exponential $e^{i k \cdot X}$ give $\left\langle: \prod_{j} e^{i k_{j} \cdot X_{j}}:\right\rangle$ $=\prod_{i<j}\left|\chi_{i j}\right|^{1 / 2 k_{i} \cdot k_{j}}$ where we have set $\alpha^{\prime}=\frac{1}{2}$ and

$\chi_{i j} \equiv \chi\left(z_{i j}, \tau\right)=2 \pi e^{-\pi\left(\operatorname{Im} z_{i j}\right)^{2} / \operatorname{Im} \tau_{2}}\left|\frac{\vartheta_{1}\left(z_{i j}, \tau\right)}{\vartheta_{1}^{\prime}(0, \tau)}\right|$

which is related to the bosonic Green function on the torus by $G_{i j}^{B}=-\frac{1}{4} \ln \left|\chi_{i j}\right|^{2}$. To get the whole kinematic structure of $\sqrt{g} R$ as in expression (2), one also has to take into account contributions coming from the $\mathcal{O}\left(k^{4}\right)$ terms by a "pinched off" integration [13-15]. These terms arise in the limiting case $z_{i j} \rightarrow 0$, for which we get

$$
\begin{aligned}
& \left|\chi_{i j}\right|^{2} \rightarrow\left|z_{i j}\right|^{2}, \\
& \bar{\partial} G_{i j}^{B} \rightarrow-\frac{1}{4 \bar{z}_{i j}}, \quad \partial G_{i j}^{B} \rightarrow-\frac{1}{4 z_{i j}}
\end{aligned}
$$

The world-sheet integral over the region $\left|z_{i j}\right|<\epsilon$ then yields a pole in $k_{i} \cdot k_{j}$, after analytic continuation:

$\int_{\left|z_{i j}\right|<\epsilon} d^{2} z_{i j} \frac{\left|z_{i j}\right|^{1 / 2 k_{i} \cdot k_{j}}}{16\left|z_{i j}\right|^{2}} \simeq \frac{4 \pi}{16 k_{i} \cdot k_{j}}$.

where we assumed that $\frac{1}{2}\left|k_{i} \cdot k_{j}\right| \ll\left|\frac{1}{\ln \epsilon}\right|$. After performing one world-shect integration we get the following $\mathcal{O}\left(k^{2}\right)$ expression: 


$$
\begin{aligned}
\int & \left.\prod_{i=1}^{3} d^{2} z_{i}\left\langle V_{g}^{(0)}\left(z_{i}, \bar{z}_{i}\right)\right\rangle\right|_{\mathcal{O}\left(k^{2}\right)} \\
& \sim \int d^{2} z_{1} \int d^{2} z_{2}\left(G_{23}^{F}\right)^{2} \bar{\partial}^{2} G_{12}^{B} \\
& \times\left[\left(k_{2} \epsilon^{1} k_{2}\right)\left(\epsilon^{2} \epsilon^{3}\right)+2\left(k_{3} \epsilon^{2} \epsilon^{3} \epsilon^{1} k_{2}\right)\right] \\
& + \text { cyclic perm }
\end{aligned}
$$

where $G_{i j}^{F}=G^{F}\left(z_{i j}\right)$ and

$$
G^{F}(z)=\frac{1}{4} \frac{\vartheta_{1}^{\prime}(0, \tau) \vartheta_{\alpha}(z, \tau)}{\vartheta_{\alpha}(0, \tau) \vartheta_{1}(z, \tau)}
$$

is the fermionic Green function on the torus. If we use the fact that $\left(G^{F}\right)^{2}$ can be expressed as

$$
\begin{aligned}
& \left(G^{F}(z)\right)^{2}=\frac{1}{16}\left(-\partial^{2} \ln \vartheta_{1}(z, \tau)\right. \\
& \left.\quad+4 \pi i \partial_{\tau} \ln \eta(\tau)-e_{\alpha}\right)
\end{aligned}
$$

where $e_{\alpha}=-4 \pi i \partial_{\tau} \ln Z_{\psi}$, we can replace $\left(G^{F}\right)^{2}$ by the spin dependent part $\left(-\frac{e_{\alpha}}{16}\right)$ because the other terms of $\left(G^{F}\right)^{2}$ will yield a zero result for the amplitude after summing over even spin structures. Therefore, there remains only one world-sheet integral to be done. But since

$$
\int d^{2} z \bar{\partial} G^{B}=\int d^{2} z \bar{\partial}^{2} G^{B}=0
$$

we find that the one-loop coupling in front of $\sqrt{g} R$ vanishes. This means that there is no renormalization of Newton's constant for heterotic strings in $d=4$, a fact which was also noted in [1] and [16], whereas for type II strings in $d=4$ it does get renormalized at one-loop string level, because the fermionic zero modes can be saturated by contracting left- and rightmoving fermions [16].

We now turn to the curvature squared terms and calculate the $\mathcal{O}\left(k^{4}\right)$ terms of the one-loop 4-graviton amplitude for a $d=4$ heterotic string. By doing so, we hope to determine whether or not the coefficients of the curvature squared terms of the one-loop effective action can be uniquely fixed. The four-graviton vertex correlation function is

$$
\begin{aligned}
& \left\langle\prod_{i=1}^{4} V_{g}^{(0)}\left(z_{i}, \bar{z}_{i}\right)\right\rangle=\prod_{i<j}\left|\chi_{i j}\right|^{1 / 2 k_{i} \cdot k_{j}} \\
& \quad \times(X(k, \epsilon, z)+Y(k, \epsilon, z)+Z(k, \epsilon, z))
\end{aligned}
$$

where $X(k, \epsilon, z), Y(k, \epsilon, z)$ and $Z(k, \epsilon, z)$ are polynomials of $k, \epsilon$ and bosonic- and fermionic Green functions, including a 4-, 6- and 8-fermion correlation function, respectively.

If we concentrate on $\mathcal{O}\left(k^{4}\right)$ contributions, we also have to expand the contractions of the exponential

$$
\begin{gathered}
\prod_{i<j}\left|\chi_{i j}\right|^{1 / 2 k_{i} \cdot k_{j}}=1-\sum_{i<j} k_{i} \cdot k_{j} G_{i j}^{B} \\
+\frac{1}{2}\left(\sum_{i<j} k_{i} \cdot k_{j} G_{i j}^{B}\right)^{2}-\ldots
\end{gathered}
$$

which is valid only if $\left|z_{i j}\right|>\epsilon$ because of the singularity of $G^{B}$ at the origin. For $z_{i j} \rightarrow 0$ one has to use the pinched off integration.

Performing the world-sheet integrals one realizes that many of the integrals give a zero result because of (17). Further, integrals such as $\int d^{2} z_{1} \bar{\partial}_{1}^{2} G_{12}^{B} \operatorname{cs}\left(2 K z_{12}\right) \operatorname{cs}\left(2 K z_{13}\right)$ also vanish as a result of the (quasi) periodicity of the Jacobi elliptic function $\operatorname{cs}(2 K z)$, where $K=\frac{\pi}{2} \vartheta_{3}^{2}(0, \tau)$ and which is defined as

$\operatorname{cs}(2 K z)=\frac{\vartheta_{2}(z, \tau) \vartheta_{4}(0, \tau)}{\vartheta_{1}(z, \tau) \vartheta_{3}(0, \tau)}$

So $\operatorname{cs}(2 K z)$ is related to the fermionic Green function for $\alpha=2$. Finally, we find that the $E_{1}$ and $E_{2}$ dependent part of the one-loop string amplitude is

$$
\begin{aligned}
& \mathcal{A}_{4 g}^{\text {even }}=-\frac{3}{2} g_{s}^{4} K_{4 g}^{1 \text {-loop }} \sum_{s \text { even }}(-)^{s_{1}+s_{2}} \int \frac{d^{2} \tau}{\tau_{2}} Z(\tau, \bar{\tau}, s) \\
& \times \int \prod_{i=1}^{4} d^{2} z_{i}\left(G_{34}^{F}\right)^{2} v^{2}\left(\bar{\partial} G_{34}^{B}\right)^{2}
\end{aligned}
$$

where $v=\frac{\pi}{4 \tau_{2}}$ and $K_{4 g}^{1 \text {-loop }}=\left[-s^{2} E_{1}+\left(2 s^{2}+u^{2}\right) E_{2}\right]$. The remaining world-sheet integral is given by the following result

$$
\begin{aligned}
& \int d^{2} z\left(\bar{\partial} G^{B}(z)\right)^{2}=-\frac{i \pi \tau_{2}}{8} \partial_{\bar{\tau}} \ln \left(\tau_{2} \bar{\eta}^{2}\right) \\
& =-\frac{\tau_{2}}{16} \hat{G}_{2}(\bar{\tau})
\end{aligned}
$$

where we used the heat equation and periodicity of $\vartheta_{1}(z, \tau)$, and the Eisenstein function of weight 2, $\hat{G}_{2}(\tau)=G_{2}(\tau)-\frac{\pi}{\ln \tau}$. Thus we get for the amplitude 


$$
\begin{aligned}
\mathcal{A}_{4 g}^{\text {even }} & =-\frac{6 i}{\pi}\left(\frac{g_{s}}{16}\right)^{4} K_{4 g}^{1-\text { loop }} \\
& \times \sum_{s \text { even }}(-)^{s_{1}+s_{2}} \int \frac{d^{2} \tau}{\tau_{2}} \frac{1}{|\eta(\tau)|^{4}} Z_{\text {int }} \partial_{\tau} Z_{\psi} \hat{G}_{2}(\bar{\tau})
\end{aligned}
$$

Note that $K_{4 g}^{1 \text {-loop }} \neq K_{4 g}^{\text {tree }}$. Thus either the one-loop corrections to the effective action are not proportional to the tree-level effective action, or the one-loop string amplitude contains different field theory subtractions.

The $\tau$ integral can be performed after fixing the internal sector, as, for example, a symmetric orbifold. This will be done after the field theory subtraction.

\section{Field theory comparison}

In the previous section, we computed 4-point string amplitudes and obtained particular kinematic structures for the $\mathcal{O}\left(k^{4}\right)$ parts. As we already mentioned, no combination of curvature squared terms that are expanded to the order $h^{4}$ can reproduce either $K_{4 g}^{\text {tree }}$ or $K_{4 g}^{1-\text { loop }}$. The way to proceed is to take the Lagrangian in (1), calculate all possible field theory contact and exchange graphs that contribute to $\mathcal{O}\left(k^{4}\right)$, and subtract them from the string amplitude expression to get the 1PI effective action.

To do this wc introduce $\bar{h}_{\mu \nu}$ as an off-shell graviton field. That is, unlike $h_{\mu \nu}$ which is constrained to satisfy the equation of motion, $\bar{h}_{\mu \nu}$, is an arbitrary fluctuation. We determine the vertices for two on-shell and one offshell field. Expanding the scalar curvature $\left.\sqrt{g} R\right|_{h^{2} \bar{h}}=$ $\bar{h}_{\mu \nu} V_{R}$ we get the vertex

$$
\begin{aligned}
V_{R} & =\frac{3}{8} \eta^{\mu \nu}\left(h,{ }_{\rho} h^{\rho}{ }^{\rho}\right)-\frac{1}{4}\left(h_{,}^{\mu} h^{\nu}\right)-\frac{1}{2}\left(h h^{\mu \nu}\right) \\
& -\frac{1}{2} h^{\mu \alpha}{ }_{, \rho} h^{\nu}{ }_{\alpha}{ }^{\rho}+\ldots
\end{aligned}
$$

For the moment we are only interested in terms having the form $\left(h,{ }^{\mu} h,{ }^{\nu}\right),\left(h,{ }^{\mu \nu} h\right), h^{\mu \alpha}{ }_{, \rho} h_{\alpha}^{\nu},{ }^{\rho}$ and $\left(h, \alpha,,^{\alpha}\right.$ ) because in exchange graphs it will be these terms that contribute to those kinematic terms depending on $E_{1}$ and $E_{2}$, which we picked out for the comparison with the 4-graviton string amplitude. The vertex for $R_{\mu \rho \nu}{ }^{\sigma} R^{\mu \rho \nu}{ }_{\sigma}$ can be obtained by taking

$$
\left.\sqrt{g} R_{\mu \rho \nu}{ }^{\sigma} R_{\sigma}^{\mu \rho \nu}\right|_{h^{2} \bar{h}}=\bar{h}_{\mu \nu} V_{\left(R_{\mu \rho \nu \sigma}\right)^{2}}^{\mu \nu},
$$

the vertex for $R_{\mu \nu} R^{\mu \nu}$ by

$$
\left.\sqrt{g} R_{\mu \nu} R^{\mu \nu}\right|_{h^{2} \bar{h}}=\bar{h}_{\mu \nu} V_{\left(R_{\mu \nu}\right)^{2}}^{\mu \nu}
$$

and the $R^{2}$ vertex by

$$
\left.\sqrt{g} R^{2}\right|_{h^{2} \bar{h}}=\bar{h}_{\mu \nu} V_{\left(R^{2}\right)}^{\mu \nu} \text {. }
$$

We find

$$
\begin{aligned}
& V_{\left(R_{\mu \rho \sigma}\right)^{2}}^{\mu \nu}=\frac{1}{2} \eta^{\mu \nu}\left(h^{\rho \sigma}{ }^{\rho} h_{\rho \sigma \sigma}\right)-5\left(h^{\mu \alpha}{ }_{\rho \sigma} h^{\nu}{ }_{\alpha}{ }^{\rho \sigma}\right) \\
& -\left(h{ }^{\mu \nu} \rho h{ }^{\mu}\right)+\ldots \\
& V_{\left(R_{\mu \nu}\right)^{2}}^{\mu \nu}=\left(h{ }^{\mu \rho} h,{ }_{\rho}\right)+\frac{1}{2}\left(h{ }^{\rho} h{ }^{\mu \nu}{ }_{\rho}\right) \\
& -\left(h^{\mu \alpha}{ }_{, \rho \sigma} h_{\alpha}{ }_{\alpha}{ }^{\rho \sigma}\right)+\ldots \\
& V_{\left(R^{2}\right)}^{\mu \nu}=3\left(h{ }^{\mu \rho} h{ }^{\mu}{ }_{\rho}\right)+3\left(h,{ }^{\rho} h,{ }^{\mu \nu}{ }_{\rho}\right) \\
& -3 \eta^{\mu \nu}\left(h^{\alpha \rho} h_{\alpha \rho \rho}\right)+\ldots
\end{aligned}
$$

Having defined the vertices, we now introduce the internal propagator for the exchange graphs. Expanding (1) to quadratic order and inverting the kernel, one finds the following propagator:

$$
\begin{gathered}
D_{\mu \nu \rho \sigma}=-\left\{\frac{P_{\mu \nu \rho \sigma}^{(2)}}{k^{2}\left(1+k^{2}(b+4 a)\right)}\right. \\
\left.-\frac{P_{\mu \nu \rho \sigma}^{(0)}}{2 k^{2}\left(1+2 k^{2}(-3 a-b-3 c)\right)}\right\}
\end{gathered}
$$

where $P_{\mu \nu \rho \sigma}^{(2)}$ and $P_{\mu \nu \rho \sigma}^{(0)}$ are transverse projectors for spin-2 and spin- 0 , respectively

$$
\begin{aligned}
& P_{\mu \nu \rho \sigma}^{(2)}=\frac{1}{2}\left(\theta_{\mu \rho} \theta_{\nu \sigma}+\theta_{\mu \sigma} \theta_{\nu \rho}\right)-P_{\mu \nu \rho \sigma}^{(0)} \\
& P_{\mu \nu \rho \sigma}^{(0)}=\frac{1}{3} \theta_{\mu \nu} \theta_{\mu \nu}
\end{aligned}
$$

where $\theta_{\mu \nu}=\eta_{\mu \nu}-\omega_{\mu \nu}$ and $\omega_{\mu \nu}=\frac{\partial_{\mu} \partial_{\nu}}{\square}$. We expand the propagator for small momenta and get $D_{\mu \nu \rho \sigma}=$ $\Pi_{\mu \nu \rho \sigma}+\tilde{\Pi}_{\mu \nu \rho \sigma}$, where the first term is the usual graviton propagator

$\Pi_{\mu \nu \rho \sigma}=-\frac{1}{k^{2}}\left(P_{\mu \nu \rho \sigma}^{(2)}-\frac{1}{2} P_{\mu \nu \rho \sigma}^{(0)}\right)$

and the second terms is a correction to the graviton propagator:

$$
\tilde{\Pi}_{\mu \nu \rho \sigma}=(b+4 a) P_{\mu \nu \rho \sigma}^{(2)}+(a+b+3 c) P_{\mu \nu \rho \sigma}^{(0)} .
$$

We can now calculate the field theory exchange graphs using $\Pi$ and $\tilde{\Pi}$ as internal propagators. The 


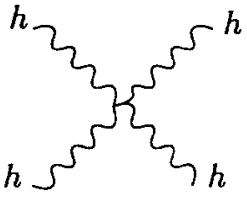

Fig. 1. Tree-level contact graph: $Q$.

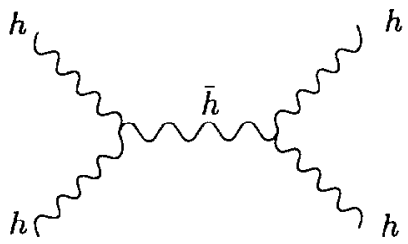

Fig. 2. Tree-level exchange graph: $V_{q} \Pi V_{R}$ and $V_{R} \tilde{\Pi} V_{R}$.

four-graviton tree-level amplitude is reported by a treelevel contact graph (Fig. 1) and tree-level exchange graphs (Fig. 2).

The fourth-order contribution to the tree level contact term is given by

$$
\begin{aligned}
Q & =\left.\sqrt{g}\left\{a R_{\mu \rho \nu}{ }^{\sigma} R^{\mu \rho \nu}{ }_{\sigma}+b R_{\mu \nu} R^{\mu \nu}+c R^{2}\right\}\right|_{h^{4}} \\
& =s^{2} E_{1}\left(-\frac{a}{4}+\frac{b}{8}+\frac{9}{16} c\right)+t^{2} E_{1}\left(\frac{a}{8}+\frac{b}{16}\right) \\
& +s^{2} E_{2}\left(\frac{3}{2} a+\frac{b}{4}\right)+\frac{a}{8} u^{2} E_{2}+\ldots
\end{aligned}
$$

The exchange graph gives rise to two terms of the order $\mathcal{O}\left(k^{4}\right)$. The first one contains the corrected propagator $\tilde{\Pi}$, giving, isolating the $E_{1}$ and $E_{2}$ terms,

$$
\begin{gathered}
V_{R}^{\mu \nu} \tilde{\Pi}_{\mu \nu \alpha \beta} V_{R}^{\alpha \beta}=s^{2} E_{1}\left(-\frac{a}{16}+\frac{b}{8}+\frac{9}{16} c\right) \\
+t^{2} E_{1} \frac{b+4 a}{16}+s^{2} E_{2} \frac{b+4 a}{4}+\ldots
\end{gathered}
$$

and the second exchange diagram has the usual graviton propagator as the internal propagator

$$
\begin{aligned}
& V_{q}^{\mu \nu} \Pi_{\mu \nu \alpha \beta} V_{R}^{\alpha \beta}=s^{2} E_{1}\left(\frac{a}{4}-\frac{b}{4}-\frac{9}{8} c\right) \\
& \quad+t^{2} E_{1}\left(-\frac{a}{4}-\frac{b}{8}\right)+s^{2} E_{2}\left(-\frac{5}{2} a-\frac{b}{2}\right)+\ldots
\end{aligned}
$$

where $V_{q}^{\mu \nu}=a V_{\left(R_{\mu \nu \nu \sigma}\right)^{2}}^{\mu \nu}+b V_{\left(R_{\mu v}\right)^{2}}^{\mu \nu}+c V_{\left(R^{2}\right)}^{\mu \nu}$ is the vertex for the quadratic curvature terms. If we add up ev-

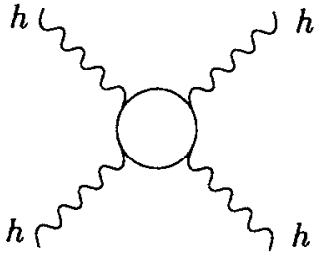

Fig. 3. 1-loop-level contact graph: $\left(\Delta_{\mathrm{gr}} Q\right)$.

erything, retaining only the $E_{1}$ and $E_{2}$ terms, we get the following result,

$$
\begin{aligned}
Q & +V_{R}^{\mu \nu} \tilde{\Pi}_{\mu \nu \alpha \beta} V_{R}^{\alpha \beta}+V_{q}^{\mu \nu} \Pi_{\mu \nu \alpha \beta} V_{R}^{\alpha \beta} \\
& =\frac{a}{8}\left\{\left(\frac{1}{2} s^{2}+t^{2}\right) E_{1}+u^{2} E_{2}\right\}=\frac{a}{8} K_{4 g}^{\text {tree }}
\end{aligned}
$$

This result tells us that tree-level string amplitudes are reproduced by the Riemann squared term only and that the coefficients $b$ and $c$, which are associated with the Ricci squared and the scalar curvature squared term respectively, do not appear. They cancel during the summation over contact and exchange diagrams. It follows that the on-shell string amplitudes do not fix a particular combination of curvature squared terms.

Before discussing the consequences of this result, let us consider the situation for the genus-one case. Similar to tree level, there is also one contact term (Fig. 3), which gives $\left(\Delta_{\mathrm{gr}} Q\right)$, where

$$
\begin{aligned}
\Delta_{\mathrm{gr}} & =\frac{1}{i \pi} \sum_{s \text { even }}(-)^{s_{1}+s_{2}} \int \frac{d^{2} \tau}{\tau_{2}} \frac{1}{|\eta(\tau)|^{4}} \\
& \times Z_{\text {int }} \partial_{\tau} Z_{\psi} \hat{G}_{2}(\bar{\tau})
\end{aligned}
$$

is replacing the blob representing one-loop 1PI processes.

There is no wavefunction renormalization of onshell external legs, because we have $\left.\sqrt{g} R\right|_{h \bar{h}}=0$, $\left.\sqrt{g} R_{\mu \rho \nu}{ }^{\sigma} R^{\mu \rho \nu}{ }_{\sigma}\right|_{h \bar{h}}=0$ and the same is true for the Ricci squared and the scalar curvature squared term. Hence, Fig. 4 does not contribute to the curvature squared term in the effective action.

Furthermore, we have one contribution from Fig. 5 which is given by $\left(\Delta_{\mathrm{gr}} \tilde{V}_{q}^{\mu \nu}\right) \Pi_{\mu \nu \alpha \beta} V_{R}^{\alpha \beta}$, where the factor $\frac{1}{2}$ reflects the fact that there is no renormalization of Newton's constant and $\tilde{V}_{q}^{\mu \nu}$ stands for the one-loop curvature squared term with one-loop coefficients, $a$, $b$ and $c$. 


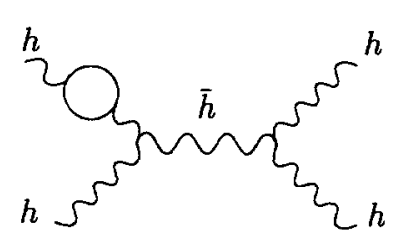

Fig. 4. Wavefunction renormalization of external legs.

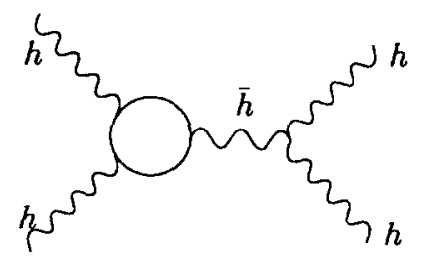

Fig. 5. Contribution from: $\left(\Delta_{\mathrm{gr}} \tilde{V}_{q}\right) \Pi V_{R}$.

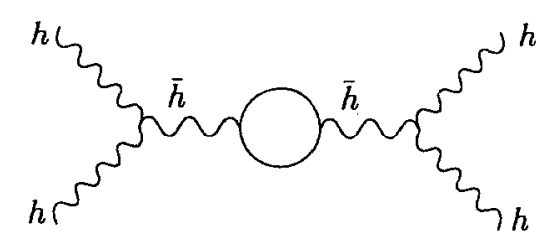

Fig. 6. Contributions from: $V_{q} \Pi\left(\Delta_{\mathrm{gr}} \bar{V}_{R}\right) \Pi V_{R}$ and $V_{R} \Pi\left(\Delta_{\mathrm{g}} \bar{V}_{R}\right) \tilde{\Pi} V_{R}$ and $V_{R} \Pi\left(\Delta_{\mathrm{gr}} \bar{V}_{q}\right) \Pi V_{R}$.

Finally there are three terms of the order $\mathcal{O}\left(k^{4}\right)$ coming from a diagram with an internal blob like Fig. 6. Before giving the explicit expression of these terms, we compute the kinematic expression for the field theory two-point function with two external off shell legs $\bar{h}$ for various curvature terms. The expansion of $\left.\sqrt{g} R\right|_{\bar{h}^{2}}$ gives $\left(\bar{V}_{R}\right)_{\mu \nu \rho \sigma}=\frac{s}{4} \Pi_{\mu \nu \rho \sigma}$ and $\left.\sqrt{g}\left\{a R_{\mu \rho \nu}{ }^{\sigma} R^{\mu \rho \nu}{ }_{\sigma}+b R_{\mu \nu} R^{\mu \nu}+c R^{2}\right\}\right|_{h^{2}}$ leads to $\left(\bar{V}_{q}\right)_{\mu \nu \rho \sigma}=\frac{s^{2}}{4} \tilde{\Pi}_{\mu \nu \rho \sigma}$. The term

$V_{q}^{\alpha \beta} \Pi_{\alpha \beta \mu \nu}\left(\Delta_{\mathrm{gr}} \bar{V}_{R}^{\mu \nu \gamma \delta}\right) \Pi_{\gamma \delta \rho \sigma} V_{R}^{\rho \sigma}$

does not give any contribution to the one-loop effective action because of the tree level vertex $V_{q}^{\alpha \beta}$ depends on tree-level coefficients. There are two more $\mathcal{O}\left(k^{4}\right)$ contributions, namely

$$
\begin{aligned}
& V_{R}^{\alpha \beta} \Pi_{\alpha \beta \mu \nu}\left(\Delta_{\mathrm{gr}} \bar{V}_{R}^{\mu \nu \gamma \delta}\right) \tilde{\Pi}_{\gamma \delta \rho \sigma} V_{R}^{\rho \sigma} \\
& \quad=-\frac{\Delta_{\mathrm{gr}}}{4}\left\{(b+4 a)\left(V_{R}\right)_{\rho \sigma} V_{R}^{\rho \sigma}-\frac{(b+5 a-c)}{4} V_{R}^{2}\right\}
\end{aligned}
$$

$$
\begin{aligned}
& V_{R}^{\alpha \beta} \Pi_{\alpha \beta \mu \nu}\left(\Delta_{\mathrm{gT}} \bar{V}_{q}^{\mu \nu \gamma \delta}\right) \Pi_{\gamma \delta \rho \sigma} V_{R}^{\rho \sigma} \\
& \quad=\frac{\Delta_{\mathrm{gr}}}{4}\left\{(b+4 a)\left(V_{R}\right)_{\rho \sigma} V_{R}^{\rho \sigma}-\frac{(b+5 a-c)}{4} V_{R}^{2}\right\}
\end{aligned}
$$

These two exchange graphs cancel against each other. Adding up the exchange graphs with one blob at the three point vertex and the contact term, we get

$$
\begin{aligned}
& \left(\Delta_{\mathrm{gr}} Q\right)+\frac{1}{2}\left(\Delta_{\mathrm{gr}} \tilde{V}_{q}^{\alpha \beta}\right) \Pi_{\alpha \beta \rho \sigma} V_{R}^{\rho \sigma} \\
& \quad=\Delta_{\mathrm{gr}}\left\{\frac{a}{8}\left(-s^{2} E_{1}+\left(2 s^{2}+u^{2}\right) E_{2}\right)\right\} \\
& \quad=\Delta_{\mathrm{gr}} \frac{a}{8} K_{4 g}^{1-\text { loop }}
\end{aligned}
$$

The only one-loop coefficient that survives the field theory subtractions is, as in the tree-level case, $a$, the cocfficicnt of $\sqrt{g} R_{\mu \rho \nu}{ }^{\sigma} R^{\mu \rho \nu}{ }_{\sigma}$. All other coefficients cancel against each other. Therefore, at the genus-one level, as in the genus-zero case, the coefficients $b$ and $c$ are not determined. They remain completely ambiguous. Thus, for example, the topological Gauss-Bonnet (GB) term, which has $b=-4 a$ and $c=a$, and the $C_{\mu \rho \nu}{ }^{\sigma} C^{\mu \rho \nu}{ }_{\sigma}$ term, with $b=-2 a$ and $c=-\frac{1}{3}$, produce the same kinematic expression in the string $S$ matrix.

Thus we learn that on-shell string amplitudes can only fix the coefficient of $\sqrt{g} R_{\mu \rho \nu}{ }^{\sigma} R^{\mu \rho \nu}{ }_{\sigma}$ as an unambiguous coefficient, whereas the coefficients of $\sqrt{g} R_{\mu \nu} R^{\mu \nu}$ and $\sqrt{g} R^{2}$ are completely ambiguous. They cancel when considering both contact and exchange graphs and the situation for the genus- 1 case is exactly the same as for the genus- 0 case. We expect this situation to continue at any higher genus as well. We conclude that one could use any one of a continuously infinite set of curvature squared terms, indexed by arbitrary coefficients $b$ and $c$, in the string effective action, because the string $S$ matrix would see no difference between them. This means that field redefinitions are a symmetry of the perturbative string $S$ matrix. This is precisely the content of the equivalence theorem [18-21], which claims that, for example, redefining the metric $\tilde{g}_{\mu \nu}=g_{\mu \nu}+c_{1} R_{\mu \nu}+c_{2} g_{\mu \nu} R$ will change the coefficients $b$ and $c$ of the effective Lagrangian $\mathcal{L}$ so that $\delta a=0, \delta b=c_{1}$ and $\delta c=-\frac{c_{1}}{2}-c_{2}$, but does not change the $S$ matrix; that is $S=\tilde{S}$. Our results explicitly verify previous results on field redefinitions. We also show exactly how this ambiguity 
arises within individual genus-zero and genus-one amplitudes.

It is important to emphasize that an effective action with only a GB term, for example, is physically different from an effective action involving $R^{2}$ or $C_{\mu \rho \nu}{ }^{\sigma} C^{\mu \rho \nu}{ }_{\sigma}$. In the pure GB case, the theory represents only a spin- 2 graviton whereas the $R^{2}$ case has, in addition to the spin- 2 graviton, an additional ghost-free scalar degree of freedom. Similarly, a $C_{\mu \rho \nu}{ }^{\sigma} C^{\mu \rho \nu}{ }_{\sigma}$ term in the effective action would add a symmetric tensor of ghost-like fields to the spectrum. These effective actions are physically inequivalent, and it is not possible to change one into the other by a field redefinition or any other means. It is therefore of importance to know the complete higher-derivative structure of the superstring effective action. Unfortunately, the results of this paper show that it is not possible to determine this structure by computing on-shell, $S$-matrix string amplitudes. Such computations can always be reproduced by an infinite number of effective actions indexed by coefficients $b$ and $c$, and this ambiguity can never be resolved in this manner. This does not mean, however, that one can use field redefinitions to choose some convenient higher-derivative action, such as pure GB. It simply means that one would have to perform some kind of off-shell superstring calculation, such as in string field theory, to exactly determine the structure of the higher-derivative gravitational terms.

\section{Gravitational threshold corrections for symmetric orbifolds}

Moduli-dependent threshold corrections have been discussed in $[1,2,6]$, by calculating a $C P$ odd amplitude. Since we are interested in the kinematic structure of the $C P$ even part of the effective action corresponding to moduli dependent gravitational corrections, we now want to calculate the $C P$ even string amplitude including four graviton vertex operators and one modulus vertex

$V_{T}^{(0)}(z, \bar{z})=: v_{I J} \partial X^{I}\left(\partial X^{J}+i k \cdot \psi \psi^{J}\right) e^{i k \cdot X}:$

where $v_{I J}=\partial_{T}\left(G_{I J}+B_{I J}\right), G_{I J}$ is the metric and $B_{I J}$ the antisymmetric tensor of the six dimensional internal compact manifold. The five-point amplitude is

$$
\begin{aligned}
& \left\langle\prod_{i=1}^{4} V_{g}^{(0)}\left(z_{i}, \bar{z}_{i}\right) V_{T}^{(0)}\left(z_{5}, \bar{z}_{5}\right)\right\rangle \\
& \quad=v_{I J}\left\langle\bar{\partial} X^{I} \partial X^{J}\right\rangle\left\langle\prod_{i=1}^{4} V_{g}^{(0)}\left(z_{i}, \bar{z}_{i}\right)\right\rangle
\end{aligned}
$$

which can be computed to give the result

$$
\mathcal{A}_{4 g 1 T}^{\text {even }}=\frac{6 g_{s}^{5}}{16^{4}} v_{I J} K_{4 g}^{1-\text { loop }} \int d^{2} \tau \mathcal{B}_{\mathrm{gr}}^{I J}(\tau, \bar{\tau})
$$

where

$$
\begin{aligned}
& \mathcal{B}_{\mathrm{gr}}^{I J}(\tau, \bar{\tau})=\frac{1}{i \pi}\left\langle\bar{\partial} X^{I} \partial X^{J}\right\rangle \\
& \quad \times \sum_{s \text { even }}(-)^{s_{1}+s_{2}} \frac{1}{|\eta(\tau)|^{4}} Z_{\text {int }} \partial_{\tau} Z_{\psi} \hat{G}_{2}(\bar{\tau}) .
\end{aligned}
$$

It is only the $N=2$ sector that gives a non-vanishing contribution to $\mathcal{B}_{\mathrm{gr}}^{I J}$, because in this sector the zero modes of $X^{I}$ can be saturated.

If we write $\mathcal{B}_{\mathrm{gr}}^{I J}(\tau, \bar{\tau})=\left\langle\bar{\partial} X^{I} \partial X^{J}\right\rangle \mathcal{B}_{\mathrm{gr}}(\tau, \bar{\tau})$ we have a similar expression to the threshold corrections to gauge couplings [6]. Therefore, we can express $\mathcal{B}_{\mathrm{gr}}$ in factorized from as $\mathcal{B}_{\mathrm{gr}}=Z_{\text {torus }}(\tau, \bar{\tau}) \mathcal{C}_{\mathrm{gr}}(\bar{\tau})$, where $Z_{\text {toras }}=\sum_{p L, p_{R} \in \Gamma_{2,2}} q^{p_{L}^{2} / 2} \bar{q}^{p_{R}^{2} / 2}$ is the partition function of the zero modes of $X^{I}$ and $\Gamma_{2,2}$ is an even self-dual Lorentzian lattice. The coupling appearing in the fivepoint amplitude is thus

$$
\partial_{T} \Delta_{\mathrm{gr}}=\frac{1}{i \pi} \int \frac{d^{2} \tau}{2 \pi \tau_{2}} \partial_{T} Z_{\text {torus }} \mathcal{C}_{\mathrm{gr}}(\bar{\tau})
$$

If we apply $\partial_{\bar{T}}$ on both sides of this equation and use the fact that [1]

$\partial_{\bar{T}} \partial_{T} Z_{\text {torus }}=\frac{4 \tau_{2}}{(T+\bar{T})^{2}} \partial_{\bar{T}} \partial_{T}\left(\tau_{2} Z_{\text {torus }}\right)$

we can perform the $\tau$ integral which becomes a contour integral around the fundamental region. The only non-vanishing contribution comes from the $\tau_{2} \rightarrow \infty$ region, because of the modular invariance of the integrand. Thus we get directly the result

$\partial_{\bar{T}} \partial_{T} \Delta_{\mathrm{gr}}=\frac{b_{\mathrm{gr}}}{\pi^{2}(T+\bar{T})^{2}}$

where $b_{\mathrm{gr}}=\lim _{\tau_{2} \rightarrow \infty} \mathcal{B}_{\mathrm{gr}}$ is the trace anomaly for the $N=2$ sector. Integrating the last expression yields 
to the gravitational coupling of the curvature squared terms

$\Delta_{\mathrm{gr}}(T, \bar{T})=-\frac{b_{\mathrm{gr}}}{\pi^{2}} \ln \left(|\eta(i T)|^{4}(T+\bar{T})\right)$

If we now use the results of the last section, we know that the moduli dependent threshold correction $\Delta_{\mathrm{gr}}$ is the coefficient of $\sqrt{g} R_{\mu \rho \nu}{ }^{\sigma} R^{\mu \rho \nu}{ }_{\sigma}$. There could also be $R_{\mu \nu} R^{\mu \nu}$ or $R^{2}$ terms whose coefficients are ambiguous and cannot be determined by string amplitude calculations. Therefore, this part of the one-loop string effective Lagrangian is given by

$$
\mathcal{L}=\sqrt{g} \Delta_{\mathrm{gr}}\left(R_{\mu \rho \nu}{ }^{\sigma} R^{\mu \rho \nu}{ }_{\sigma}+b R_{\mu \nu} R^{\mu \nu}+c R^{2}\right)
$$

As discussed above, it would require some sort of offshell superstring calculation to fix the $b$ and $c$ coefficients uniquely. Expression (50) is the best that one can compute using on-shell string amplitudes.

\section{Acknowledgements}

K.F. would like to thank J. Louis and V. Kaplunovsky for useful discussions and the University of Pennsylvania for hospitality. This work was supported in part by DOE Contract DOE-AC02-76-ERO-3071 and NATO Grant CRG. 940784 and GIF - the GermanIsraeli Foundation.

\section{References}

[1] I. Antoniadis, E. Gava and K.S. Narain, Phys. Lett. B 283 (1992) 209.

[2] I. Antoniadis, K.S. Narain and T.R. Taylor, Phys. Lett. B 267 (1991) 37.
[3] A. Hindawi, B. Ovrut and D. Waldram, Two-Dimensional Higher-Derivative Supergravity and a New Mechanism for Supersymmetry Breaking, hep-th-9509174;

Four-Dimensional Higher-Derivative Supergravity and Spontaneous Supersymmetry Breaking, hep-th-9511223; Soft Supersymmetry Breaking Induced by Higher-Derivative Supergravitation in the Electroweak Standard Model, hepth-9602075.

[4] G.L. Cardoso, D. Lüst and B.A. Ovrut, Nucl. Phys. B 436 (1995) 65.

[5] V.S. Kaplunovsky, Nucl. Phys. B 307 (1988) 145.

[6] L.J. Dixon, V.S. Kaplunovsky and J. Louis, Nucl. Phys. B 355 (1991) 649.

[7] D.J. Gross and J.H. Sloan, Nucl. Phys. B 291 (1987) 41.

[8] Y. Cai and C.A. Nunez, Nucl. Phys. B 287 (1987) 279.

[9] J. Ellis, P. Jetzer and L. Mizrachi, Nucl. Phys. B 303 (1988) 1.

[10] N. Sakai and Y. Tanii, Nucl. Phys. B 287 (1987) 457.

[11] M. Abe, H. Kubota and N. Sakai, Nucl. Phys. B 306 (1988) 405.

[12] D. Lüst, S. Theisen and G. Zoupanos, Nucl. Phys. B 296 (1988) 800 .

[13] J.A. Minahan, Nucl. Phys. B 298 (1988) 36.

[14] P. Mayr and S. Stieberger, Nucl. Phys. B 412 (1994) 502.

[15] Z. Bern and D.A. Kosower, Nucl. Phys. B 321 (1989) 605.

[16] E. Kiritsis and C. Kounnas, Nucl. Phys. B 442 (1995) 472.

[17] S. Deser and A.N. Redlich, Phys. Lett. B 176 (1986) 350.

[18] R.R. Metsaev and A.A. Tseytlin, Nucl. Phys. B 293 (1987) 385; Phys. Lett. B ( 1987) 354.

[19] A.A. Tseytlin, Phys. Lett. B (1986) 92, Nucl. Phys. B 276 (1986) 391.

[20] C. Itzykson and J. Zuber, Quantum Field Theory (McGrawHill, New York, 1980).

[21] R. Haag, Local Quantum Physics (Springer-Verlag, 1992).

[22] B. Zwiebach, Phys. Lett. B 156 (1985) 315. 